\title{
P-0578 Radial pulse spectrum may be a risk marker of peripheral artery disease in patients with type 2 diabetes
}

Kuo-meng Liao ${ }^{1}$, Ying-chun Chen ${ }^{1}$, Sheng-Hung Wang ${ }^{2}$, Ming-Yie Jan², Chi-Wei Chang ${ }^{3}$

${ }^{1}$ Division of Endocrinology \& Metabolism of Zhongxiao Branch of Taipei City Hospital, Taipei, Taiwan, ROC

${ }^{2}$ Biophysics Laboratory, Institute of Physics, Academia Sinica, Taipei, Taiwan, ROC

${ }^{3}$ Graduate Institute of Biomedical Electronics and Bioinformatics, National Taiwan University, Taipei,

Taiwan, ROC

Peripheral artery disease (PAD) is an independent risk factor for increasing morbidity and mortality of cardiovascular disease, especially in diabetic patients. PAD can lead to intermittent claudication, ulceration, and more severely limb amputation. An early predictor and good health management can retard the progression of PAD and reduce the risk of cardiovascular events. The study aimed to examine whether the spectrum analysis of radial pressure wave can predict PAD in patients with Type 2 diabetes.Radial pressure wave was measured by a piezoresistive sensor in 521 (Male: 299, Female: 222) Type 2 diabetic patients with PAD $(n=103)$ and without PAD $(n=418)$. For each patient, spectrum analysis of radial pressure wave was calculated and transformed into Fourier series coefficients $\mathrm{Cn}$. The means of second (C2) harmonic of arterial pulse were significantly higher in patients with $\mathrm{PAD}(\mathrm{C} 2: 0.549 \pm 0.139)$ than in patients without $\mathrm{PAD}(\mathrm{C} 2: 0.515 \pm 0.098, \mathrm{P}<0.01$ respectively), while the means of fifth (C5) harmonic of arterial pulse were significantly lower in patients with PAD (C5: 0.124 \pm 0.039$)$ than in patients without PAD $(\mathrm{C} 5: 0.133 \pm 0.04, \mathrm{P}<0.05)$. Chi-square test proved there were associations between $\mathrm{C} 2$ and $\mathrm{PAD}(\mathrm{C} 2: \mathrm{P}<0.05)$. The risks of $\mathrm{PAD}$ in patients with diabetes were raised by the increments of $\mathrm{C} 2$ (Odds ratio: 1.82, 95\% C.I. 1.04-3.19, $\mathrm{P}<0.05$ ). Multivariable regression analysis showed $\mathrm{C} 2$, $\mathrm{C} 5$, triglyceride, and duration of diabetes were associated with $\operatorname{PAD}(\mathrm{R}=0.505, \mathrm{P}<0.05)$. This report identified the second harmonics of radial pressure wave could be one of the important predictors for early diagnosis of PAD in type 2 diabetic patients.

Patients with type 2 diabetes who underwent measurement of radial pressure wave between March 2016 and January 2017 at the Division of Endocrinology \& Metabolism of Zhongxiao Branch of Taipei City Hospital were enrolled in this study after receiving approval from the institutional review board of Taipei City Hospital (IRB number: TCHIRB-10512113-E). All the subjects signed the informed consent about the pressure wave measurement and that cross-sectional clinical data were obtained from the medical records. Measurement of radial pressure wave was performed in a quiet room at a constant temperature of 23-25. During each measurement, the patient was lying in a supine position after 10 minutes of rest and the signal was received from harmonic wave analyzer TD01C (MII-ANN Technology, Taiwan) at the sampling rate of $400 \mathrm{~Hz}$. Twelve-second sequential pulses were acquired and transformed into Fourier series coefficients for each pulse. The harmonic components are defined by the following equation: $\mathrm{C}_{\mathrm{n}}=\mathrm{A}_{\mathrm{n}} / \mathrm{A}_{0}$

This study focused on the first eleven harmonic components $(\mathrm{C} 1 \sim \mathrm{C} 11)$, where A0 is the mean value of pulse pressure and $\mathrm{An}$ is the nth coefficient of Fourier series. For each specific harmonic component, the mean value of $\mathrm{Cns}$ calculated from all pulses was used as a representative harmonic amplitude value within one measurement.

We performed two statistical analyses to assess the association between harmonics of the radial pressure wave and PAD. At first, the subjects were divided into two groups: type 2 diabetic patients with and without PAD. PAD was recognized and prescribed by the doctors in clinical practice with ankle-brachial index (ABI) less than 0.9 for either side or ABI:0.9-1.0 for both side. Variables from medical records and from the spectrum analysis of radial pressure wave were calculated in the two groups and were given as mean \pm SD (Table 1). We compared means of those functional variables of the two groups using the Student's t-test. The harmonics of radial pressure wave performed the chi-squared test to examine the independent association between the harmonics and PAD. The odds ratios for PAD with exposure to higher and lower values of those specific harmonics were assessed.

In the second, the risk of developing PAD was calculated by the multivariable linear regression model. Characteristics of radial pressure such as harmonic amplitude and phase were major screening parameters while age, gender, smoking, BMI, HBA1C, diastolic pressure, systolic pressure, TG, creatine, and duration of diabetes were included as covariates. The statistical analyses were performed using the Matlab software.The clinical data on the two groups of patients were shown in Table 1. The diabetic patients with PAD has larger mean of BMI $(\mathrm{P}<0.01)$ and triglycerides $(\mathrm{P}<0.05)$. The means of second $(\mathrm{C} 2)$ harmonic of arterial pulse were significantly higher in patients with PAD (C2:0.55 $\pm 0.14, C 5: 0.12 \pm 0.04)$ than in patients without PAD $(\mathrm{C} 2: 0.51 \pm 0.10, \mathrm{P}<0.01)$.
Table 1. Clinical characteristics of the 1137 diabetic patients enrolled in the study, divided according to the presence or absence of Peripheral artery disease.

\begin{tabular}{ccc}
\hline & $\begin{array}{c}\text { Diabetes } \\
\text { With PAD }\end{array}$ & $\begin{array}{c}\text { Diabetes } \\
\text { Without PAD }\end{array}$ \\
\hline N & 103 & 418 \\
Male (\%) & 54 & 58 \\
Age (year) & $62.1 \pm 12.7$ & $60.7 \pm 11.1$ \\
BMI (kg/m $\left.\mathbf{m}^{2}\right)$ & $29.0 \pm 7.1^{* * *}$ & $27.1 \pm 4.2$ \\
SBP(mmHg) & $129 \pm 10$ & $129 \pm 13$ \\
DBP (mmHg) & $74 \pm 8$ & $74 \pm 9$ \\
& & \\
HbA1C (\%) & $7.1 \pm 1.5$ & $7.0 \pm 1.2$ \\
LDL (mmo//L) & $86 \pm 24$ & $89 \pm 30$ \\
HDL (mmol/L) & $47.2 \pm 16.7$ & $49.8 \pm 14.5$. \\
TG (mmol/L) & $149 \pm 95^{* *}$ & $129 \pm 72$ \\
Albuminuria (mg/dL) & $333 \pm 567$ & $205 \pm 521$ \\
Duration of diabetes (years) & $12.8 \pm 9.0$ & $11.0 \pm 12.9$ \\
HR (beats/min) & $75 \pm 12$ & $74 \pm 11$ \\
Smokers (\%) & 9.4 & 5.9 \\
\hline
\end{tabular}

$\mathrm{PAD}=$ Peripheral artery disease, $\mathrm{SBP}=$ Systolic blood pressure, $\mathrm{DBP}=$ Diastolic blood pressure, $\mathrm{LDL}=$ low density lipoprotein cholesterol, $\mathrm{HDL}=$ high density lipoprotein cholesterol, $\mathrm{TG}=$ triglycerides, $\mathrm{HR}=$ heart rate.Single, double, and triple asterisks indicate that the means of variables in the group of diabetic patients with PAD differ significantly $(\mathrm{p}<0.1, \mathrm{p}<0.05$, and $\mathrm{p}<0.01$ respectively) from the group of diabetic patients without PAD using the Student's t-test.

Multivariable regression analysis showed $\mathrm{C} 2 \quad(\beta=1.68 \quad \mathrm{P}<0.05)$, triglyceride $(\beta=6.28 \mathrm{e}-4, \mathrm{P}<0.05)$, duration of diabetes $(\beta=0.01, \mathrm{P}<0.05)$ , and BMI $(\beta=0.0128, \mathrm{P}<0.01)$ had the independent effects on PAD $(\mathrm{R}=0.40, \mathrm{P}=0.06)$. In this cross-sectional study, we proposed and proved significant associations of cardiovascular markers $\mathrm{C} 2$ with PAD, after adjustment for triglyceride, duration of diabetes, and BMI. Further investigation was needed to examine whether the characteristic of radial pulses such as $\mathrm{C} 2$ and $\mathrm{C} 5$ has the ability to improve the risk assessment based on routine clinical examination.

Figure 1 Comparison of first five harmonic amplitude of radial pressure wave in diabetic patients with PAD and without PAD.

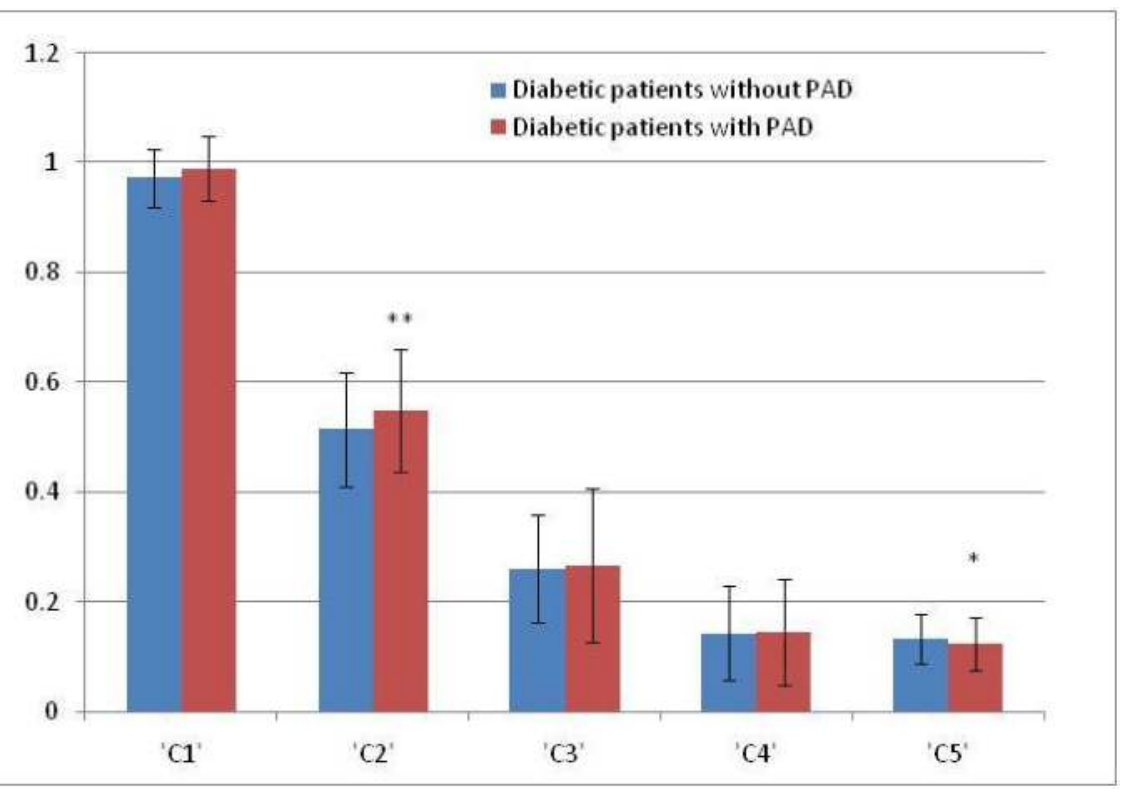

PAD : Peripheral artery disease. Single, and double asterisks indicate that the mean of $\mathrm{Cn}$ in the group of diabetic patients with PAD differs significantly ( $p<0.01, p<0.05$ respectively) from the group of diabetic patients without PAD using the Student's t-test. 